\title{
Nonlinear large scale flow in a precessing cylinder and its ability to drive dynamo action
}

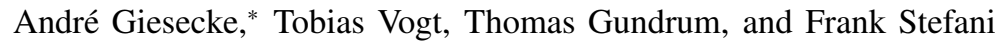 \\ Helmholtz-Zentrum Dresden-Rossendorf \\ Bautzner Landstrasse 400, D-01328 Dresden, Germany
}

(Dated: October 11, 2018)

\begin{abstract}
We have conducted experimental measurements and numerical simulations of a precession driven flow in a cylindrical cavity. The study is dedicated to the precession dynamo experiment currently under construction at Helmholtz-Zentrum Dresden-Rossendorf (HZDR) and aims at the evaluation of the hydrodynamic flow with respect to its ability to drive a dynamo. We focus on the strongly nonlinear regime in which the flow is essentially composed of the directly forced primary Kelvin mode and higher modes in terms of standing inertial waves arising from nonlinear self-interactions. We obtain an excellent agreement between experiment and simulation with regard to both, flow amplitudes and flow geometry. A peculiarity is the resonance-like emergence of an axisymmetric mode that represents a double roll structure in the meridional plane. Kinematic simulations of the magnetic field evolution induced by the time-averaged flow yield dynamo action at critical magnetic Reynolds numbers around $\mathrm{Rm}^{\mathrm{c}} \approx 430$ which is well within the range of the planned liquid sodium experiment.
\end{abstract}

PACS numbers: 91.25.Cw, 47.65.-d, 47.80.Cb, 47.20.Ky, 52.65.Kj

Keywords: dynamo, precession, rotating fluids, experiments, direct numerical simulations

Magnetic fields of celestial bodies like planets, moons or asteroids are ubiquitous in the solar system with a wide diversity of manifestations [1]. While it is undisputed that these fields are generated by conversion of mechanical energy from the flow of an electrically conductive fluid, there are various possibilities to drive the underlying fluid motions. Usually, it is assumed that the flow in liquid planetary cores is driven by thermo-compositional convection [2], yet alternative approaches invoke mechanical stirring by libration [3], tidal forcing [4] or precession [5]. In particular precession has been repeatedly proposed as source for dynamo action of the ancient lunar magnetic field [6] or the geodynamo [7]. Indeed, simulations and experiments revealed that precession may excite vigorous flows [8] which are supposed to drive a dynamo [9]. Precessional forcing has become of great interest from the experimental point of view, because it represents a natural mechanism which allows an efficient driving of conducting fluid flows on the laboratory scale without making use of propellers or pumps [10]. At HZDR a precession dynamo experiment is under development [11] which will provide a flow of liquid sodium in a cylindrical cavity with a magnetic Reynolds number of up to $\mathrm{Rm}=\Omega_{\mathrm{c}} R^{2} / \eta \approx 700$ (defined with the achievable angular velocity of the cylinder $\Omega_{\mathrm{c}}=63 \mathrm{~s}^{-1}$, the radius $R=1 \mathrm{~m}$, and the magnetic diffusivity for liquid sodium $\eta=0.09 \mathrm{~m}^{2} / \mathrm{s}$ ). The project is further motivated by previous precession experiments conducted by Gans [12] who achieved an amplification of an applied magnetic field by a factor of 3 with a device smaller by a factor of 8 and by numerical studies yielding precession driven dynamos in different geometries with a critical magnetic Reynolds number of $O\left(10^{3}\right)$ [5. 13]. However, so far numerical models of the planned experiment have not shown conclusively that the achievable magnetic Reynolds number will be sufficient to allow for dynamo action [14, 15].

In the present study we address preparatory simulations and flow measurements at a water experiment that represents a down-scaled model of the planned sodium dynamo. The re- sults provide flow patterns and amplitudes in dependence on Reynolds number $\operatorname{Re}=\Omega_{\mathrm{c}} R^{2} / v$ and on the relation of precession frequency $\Omega_{\mathrm{p}}$ to rotation frequency $\Omega_{\mathrm{c}}$, the Poincaré number Po $=\Omega_{\mathrm{p}} / \Omega_{\mathrm{c}}$. Finally, the three-dimensional velocity fields from the simulations are used in kinematic dynamo models in order to estimate parameter regimes that will be appropriate for dynamo action.

We conduct direct numerical simulations in the precession reference frame using the code SEMTEX [17]. In this frame the observer resides on the turntable following the rotation around the precession axis, thereby watching at the spinning cylinder (Fig. 17). The flow is described by the Navier Stokes equation including a time-independent term for the Coriolis force due to precession [18]:

$$
\frac{\partial}{\partial t} \boldsymbol{u}+\boldsymbol{u} \nabla \boldsymbol{u}=-\nabla P-2 \boldsymbol{\Omega}_{\mathbf{p}} \times \boldsymbol{u}+v \nabla^{2} \boldsymbol{u} .
$$

Here, $\boldsymbol{u}$ is the incompressible velocity field, $P$ the reduced pressure, $v$ the viscosity, and $\boldsymbol{\Omega}_{\mathrm{p}}$ the angular velocity of the precessional motion. The flow obeys no-slip boundary conditions for the poloidal components, $u_{r}=u_{z}=0$, whereas the azimuthal flow at the boundaries is prescribed by $u_{\varphi}=r \Omega_{\mathrm{c}}$. Fluid velocities are measured using Ultrasonic Doppler Velocimetry (UDV) which provides instantaneous profiles of the velocity component in direction of an ultrasonic beam [19, 20] oriented parallel to the cylinder axis. Four ultrasound transducers are fixed at one end cap of the cylinder (Fig. 11a) and co-rotate with the container thus providing measurements in the cylinder frame. This reference frame is well suited for flow characterization in terms of eigenmodes of rotating flows which are the solutions of the linearized inviscid version of Eq. (1). In a cylinder these solutions are inertial waves, or Kelvin modes, $\boldsymbol{U}_{j}(r, z, \varphi, t)=\tilde{\boldsymbol{u}}_{j}(r) e^{i\left(\omega_{j} t+m \varphi+k z\right)}[21,22]$ labeled by $j$ that abbreviates a triple index comprising the azimuthal wave number $m$, the axial wave number $k$, and a radial wave number index $n$. The last index counts the roots of the disper- 

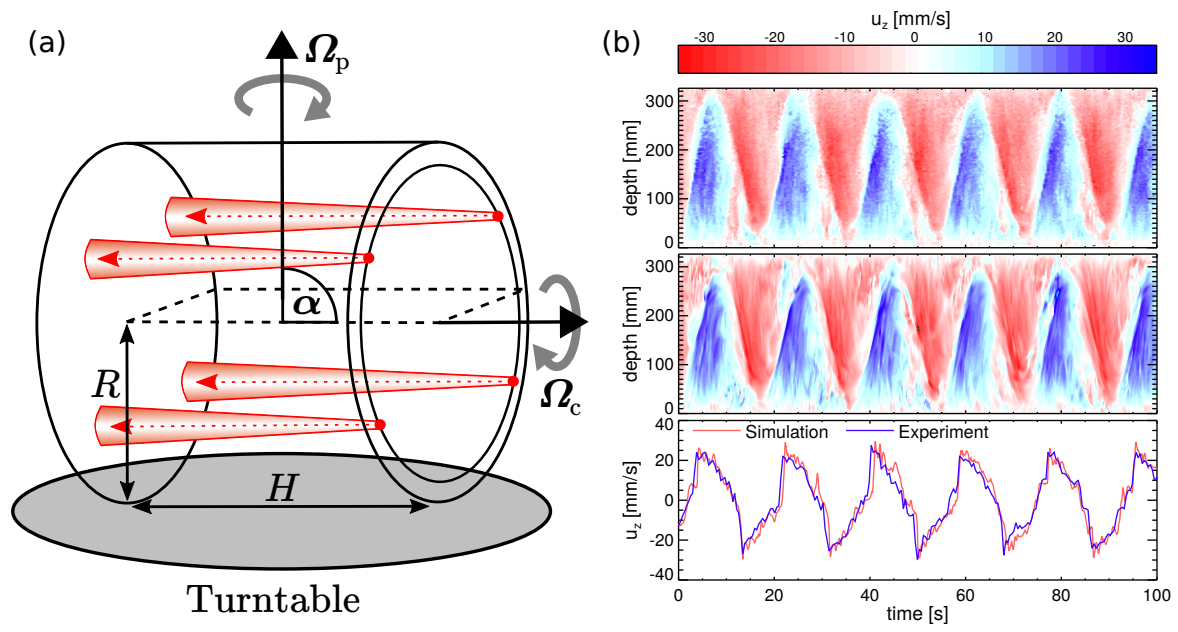

(c)

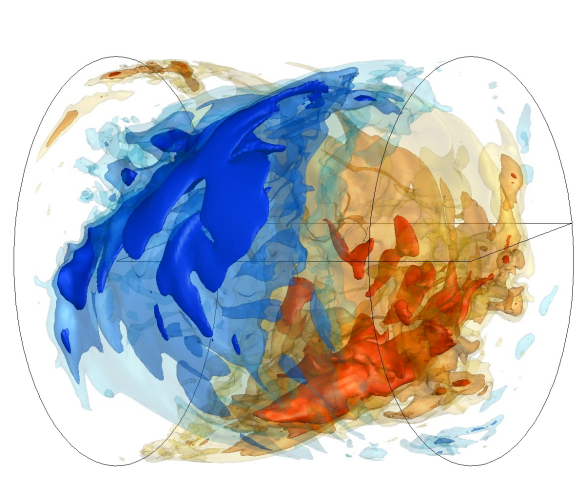

Figure 1. (a) Sketch of the experimental set-up. The red dots denote the locations of the UDV probes in the water experiment and the arrows illustrate the propagation of the ultrasound signal. (b) Temporal evolution of the axial velocity $u_{z}$ at $r=150 \mathrm{~mm}$ (top: UDV measurements, center: simulations, bottom: comparison of simulations and experiments in the equatorial plane). (c) Isosurfaces showing a snapshot of $u_{z}$ from simulations at $\mathrm{Re}=10^{4}$ and $\mathrm{Po}=0.1$. Blue (red) colors indicate flow in negative (positive) direction (see movie at [16] for temporal evolution of $u_{z}$ ).

sion relation for an inertial wave

$$
\omega_{j} \lambda_{j} J_{m-1}\left(\lambda_{j}\right)+m\left(2-\omega_{j}\right) J_{m}\left(\lambda_{j}\right)=0 \text { with } \omega_{j}=\frac{ \pm 2}{\sqrt{1+\left(\frac{\lambda_{j}}{2 k \pi}\right)^{2}}}
$$

where $J_{m}$ denotes the Bessel function of order $m$, and $\lambda_{j}$ plays the role of a radial wave number.

Precession causes a steady volume forcing with an odd symmetry with respect to the equatorial plane. Therefore the primary response of the fluid is a flow with an azimuthal wave number $m=1$ and an odd axial wave number $k$ that is stationary in the precession reference frame. If the frequency of the corresponding eigenmode $\left(\omega_{j}\right)$ exactly matches the frequency of the forcing $\left(\Omega_{\mathrm{c}}\right)$, the mode becomes resonant, and the linear inviscid approach for the computation of the amplitude fails [23]. The resonance condition delicately depends on the aspect ratio, and the primary forced mode with the simplest possible structure, i.e. $m=1, k=1$ and $n=1$ becomes resonant at $H / R=1.98982$ which is close to the geometry envisaged for our planned experiment $(H / R=2)$. In the present study the corresponding cylinder utilized in the water experiment has radius $R=163 \mathrm{~mm}$ and height $H=326 \mathrm{~mm}$, and the angle between rotation axis and precession axis is fixed at $\alpha=90^{\circ}$. Typical measurements of a single UDV probe are shown in Fig 1b (top) in terms of the axial velocity versus time and depth. The alternation of the sign of $u_{z}$ with the periodicity of $\Omega_{\mathrm{c}}$ and the asymmetry with respect to the equatorial plane illustrate the dominance of the $m=1$ component superposed by higher azimuthal modes (essentially $m=2$ ). We find a very good agreement between experiments and simulations (Fig. 11p, central and bottom panel). For sufficiently large Po, the flow is concentrated in the vicinity of the cylinder walls (Fig. 1F) and can be decomposed into few large scale modes. These modes represent standing inertial waves in the preces- sion reference frame, and time-dependent contributions only appear as weak small-scale fluctuations (see movie in supplementals [16]).

A quantitative analysis of the flow is done by decomposing axial profiles of $u_{z}$ in $k$-modes $\propto \sin (\pi z k / H)$ which is the characteristic $z$-dependence of the axial component of an inertial wave in a cylinder with height $H[23,24]$. In a second step we take the individual $k$-modes from this decomposition and apply a 2D Fourier transformation in azimuthal direction and in time which finally yields spectra that allow the identification of individual modes labeled by $(m, k)$. Typical spectra from simulations at $\operatorname{Re}=10^{4}$ and Po $=0.1$ are shown in Fig. 2 a which represents the signature of the primary forced mode $(m, k)=(1,1)$ and its first multiple $(m, k)=(2,2)$ resulting from nonlinear self-interaction. For sufficiently strong precession the spectra of all $(m, k)$-modes qualitatively look similar with one single peak at $\omega=0$ that corresponds to a standing inertial wave in the precession reference frame. The amplitudes of individual modes, estimated from spectral peaks at $\omega=0$, show that, independent of Po, the flow is always dominated by the primary forced mode $(m, k)=(1,1)$ (Fig. $2 \mathrm{p}$, blue curve). A characteristic feature is the concise maximum of the amplitude at $\mathrm{Po}^{\mathrm{c}} \approx 0.09$. Immediately following this maximum we find three phenomena that are intimately connected: a strong and abrupt reduction of the amplitude of the directly forced flow with $(m, k)=(1,1)$, a gradual increase of higher modes that originate from nonlinear self-interaction according to $(m, k) \rightarrow(2 m, 2 k)$ (Fig. 2 2 , red curve) and a sudden appearance of a non-geostrophic axisymmetric flow with $k$ even (Fig. 2 2 , green curve). The axisymmetric mode only exists with noteworthy amplitude within a rather narrow band with a width $\triangle \mathrm{Po} \sim 0.006$ (Fig. 2k). This axisymmetric mode is of interest with regard to the dynamo problem because its geometric pattern corresponds to a double roll structure (Fig. 3a) 

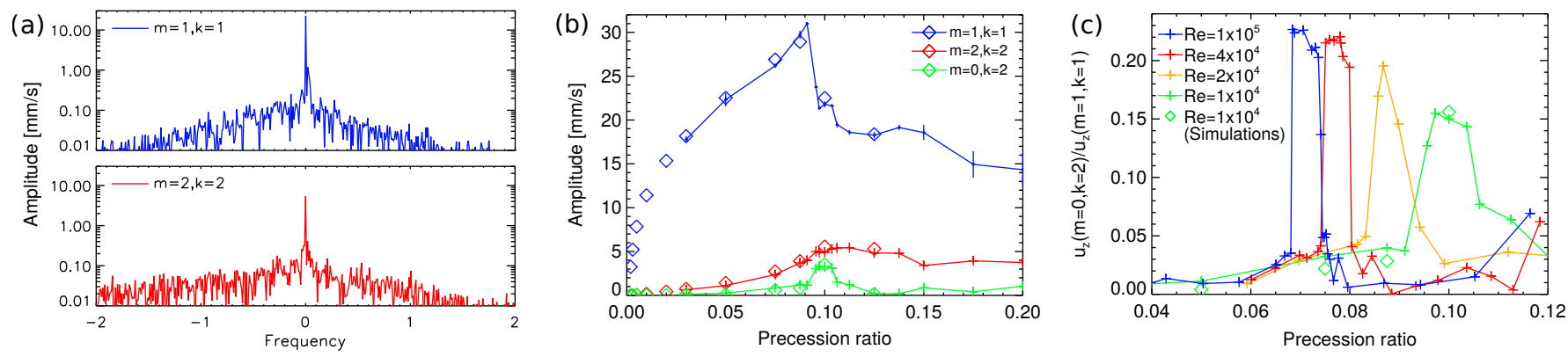

Figure 2. (a) Fourier spectra for the $(m, k)=(1,1)$ mode and for the strongest secondary mode $(m, k)=(2,2)$ from simulations at $r=150$ mm, $\operatorname{Re}=10^{4}$ and Po $=0.1$. (b) Amplitude of the time-independent part of directly forced mode $(m, k)=(1,1)$, its multiple $(m, k)=(2,2)$ and the non-geostrophic axisymmetric mode $(m, k)=(0,2)\left(\mathrm{Re}=10^{4}, r=150 \mathrm{~mm}\right)$. (c) Relative amplitude of the non-geostrophic axisymmetric mode $(m, k)=(0,2)$ with respect to $(m, k)=(1,1)$. The solid curves in (b) and (c) denote results from the water experiment, and the diamonds denote results from simulations.

(a)

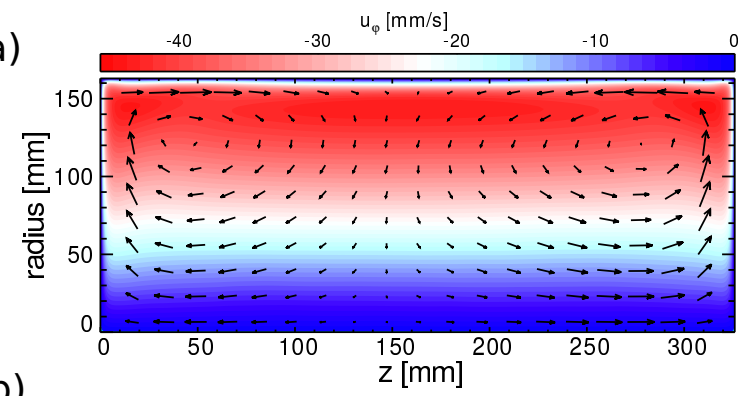

(b)

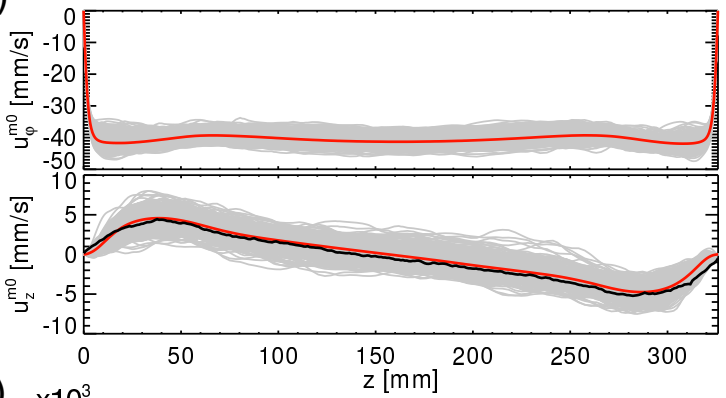

(c)

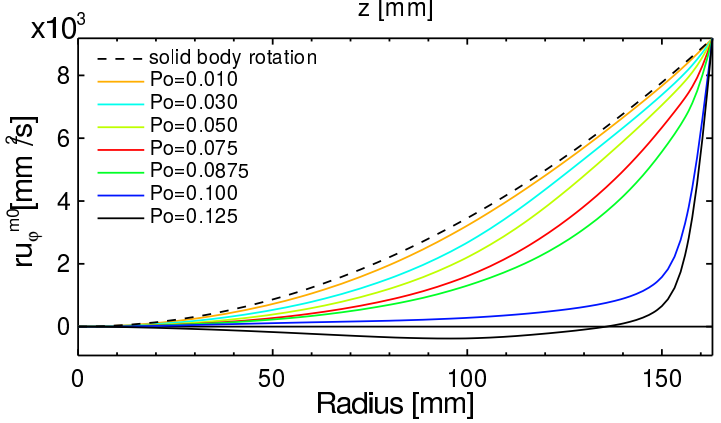

Figure 3. (a) Time-averaged axisymmetric velocity field at $\operatorname{Re}=10^{4}$ and $\mathrm{Po}=0.1$. Colors denote $u_{\varphi}$ (without solid body rotation) and arrows represent $u_{r}$ and $u_{z}$. (b) Axial profile of $u_{\varphi}$ and $u_{z}$ at $r=150 \mathrm{~mm}$. Grey curves represent temporal variations of instantaneous profiles from simulations and red curves show the time average. The black curve in the bottom panel shows the time-averaged profile obtained in the water experiment. (c) Radial profiles of the time-averaged angular momentum including solid body rotation from simulations at $\operatorname{Re}=10^{4}$. similar to the mean poloidal flow in the von-Kármán sodium dynamo in which the flow was driven by two opposite counterrotating impellers [25]. It is well known that this flow can drive a dynamo at comparatively low $\mathrm{Rm}$ [26] when the relation between toroidal and poloidal components is of order unity. However, there are further contributions to the axisymmetric flow in terms of a geostrophic azimuthal circulation (Fig. 3p) directed opposite to the solid body rotation which worsen this relation in our model.

The experiments show that the basic flow properties remain unchanged up to $\operatorname{Re}=10^{5}$ except the decrease of the critical value $\mathrm{Po}^{\mathrm{c}}$ at which the previously discussed phenomena emerge. The occurrence of the non-geostrophic axisymmetric resonance is a robust feature which does not disappear when increasing Re (Fig. 22). This mode can be excited by interacting inertial waves according to $(m, k, \omega) \rightarrow(0,2 k, 0)$ [24]. However, this is unlikely without the presence of singularities [27] so that these interactions must happen within no-slip boundary layers [28] or internal shear layers [29]. A more descriptive explanation rests upon the modification of the basic azimuthal circulation, which for sufficiently large Po compensates the bulk fluid's solid body rotation. The azimuthal fluid motion opposite to the cylinder rotation can even become so strong that eventually the Rayleigh criterion for stability of rotating fluids may be violated by developing a negative radial derivative of the angular momentum, i.e., $\frac{d}{d r}\left(u_{\varphi} r\right)<0$ (Fig. 35), immediately leading to the formation of Taylor vortices. Finally, the further increase of Po leads to the breakdown of the large scale structures into smaller scales which, at $\operatorname{Re} \approx O\left(10^{6}\right)$, corresponds to a transition into a fully turbulent flow without significant large scale contributions [32].

In the following we use the velocity fields obtained from the hydrodynamic simulations, validated by UDV flow measurements, as basis for kinematic dynamo models. We concentrate on the strongly precessing regime around $\mathrm{Po} \approx 0.1$ so that the flow is determined by standing inertial waves which makes the time-averaged velocity field appropriate for the application in kinematic simulations. The flow field is further decomposed into separate azimuthal modes $m=0,1,2$ in order to carve out 


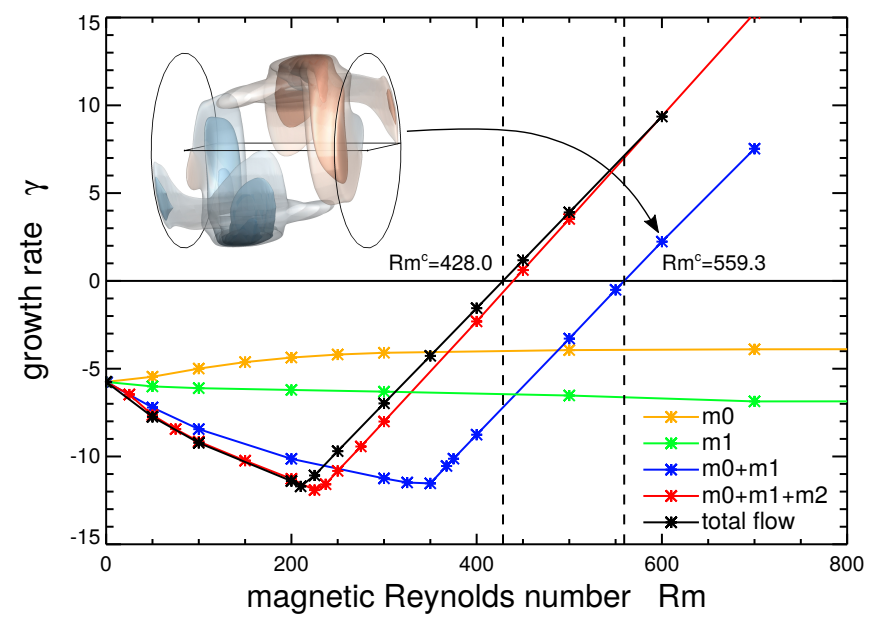

Figure 4. Growth rates for combinations of various azimuthal modes from the velocity field obtained at simulations at $\mathrm{Re}=10^{4}$ and $\mathrm{Po}=$ 0.1 . The insert drawing depicts isosurfaces of the magnetic energy density mapped with $B_{\varphi}$. In the precession frame the field structure propagates around the cylinder axis (see movie at [30]).

the impact of the individual contributions on the dynamo. The temporal evolution of the magnetic flux density $\boldsymbol{B}$ induced by a given time-averaged flow $\overline{\boldsymbol{u}}$ of a conducting liquid is determined by the induction equation

$$
\frac{\partial}{\partial t} \boldsymbol{B}=\nabla \times(\overline{\boldsymbol{u}} \times \boldsymbol{B}-\eta \nabla \times \boldsymbol{B}) .
$$

With the Ansatz $\boldsymbol{B}(\boldsymbol{r}, t)=\boldsymbol{B}_{0}(\boldsymbol{r}) e^{\sigma t}$ the solution of Eq. 3 is a linear problem with the real part of the eigenvalue $\sigma$ representing the magnetic field growth rate $\gamma$. We solve Eq. (3) numerically with pseudo-vacuum boundary conditions for the magnetic field, and the growth rates are computed from the timeevolution of the magnetic field. Except for the velocity field at Po $=0.1$, the kinematic models either show no dynamo or do so at best for magnetic Reynolds numbers far above the values that will be attainable in the planned dynamo experiment (e.g. $\mathrm{Rm}^{\mathrm{c}} \approx 5000$ for Po $=0.0875$ ). Taking the time-averaged flow field from hydrodynamic simulations at $\mathrm{Re}=10^{4}$ and Po $=0.1$, we find dynamos at much reduced $\mathrm{Rm}$. The kinematic growth rates for this particular case are shown in Fig. 4 where we distinguish five different set-ups. We find that neither the axisymmetric flow ( $m=0$, orange curve) nor the directly forced flow ( $m=1$, green curve) alone are capable of driving a dynamo. The latter was expected because the structure of the primary flow is too simplistic for dynamo action [14]. The failure of the pure axisymmetric flow to drive a dynamo confirms our previous assumption of the inappropriate relation of axisymmetric poloidal and toroidal flow components. However, when summing up both contributions we obtain dynamo action at a critical magnetic Reynolds number $\mathrm{Rm}^{\mathrm{c}} \approx 560$ (blue curve). This value decreases to $\mathrm{Rm}^{\mathrm{c}} \approx 430$ when further including the $m=2$ modes (red curve), most probably because this contribution, which is dominated by the $(m, k)=(2,2)$ mode, increases the breaking of the equatorial symmetry, which is beneficial for precession driven dynamos [5]. Other contributions with higher wave numbers are less important and no significant further reduction of $\mathrm{Rm}^{\mathrm{c}}$ is obtained when using the total time-averaged flow which yields $\mathrm{Rm}^{\mathrm{c}} \approx 428$ (black curve).

The water experiments indicate that the flow structure does not change much when increasing Re [31], albeit the corresponding decrease of $\mathrm{Po}^{\mathrm{c}}$ does not follow a simple scaling law (Fig. 2k). However, it is known from measurements of the internal pressure that the sudden drop of the $m=1$ mode, which constitutes the second criteria for $\mathrm{Po}^{\mathrm{c}}$, only weakly depends on $\operatorname{Re}$ if $\operatorname{Re} \gtrsim 5 \times 10^{5}[32]$. This is already indicated in our experiments when increasing Re from $4 \times 10^{4}$ to $10^{5}$. The width within which we observe the axisymmetric mode $(\Delta \mathrm{Po} \approx 0.006)$ corresponds nearly exactly to the width of the hysteresis found in [32] around $\operatorname{Re} \sim O\left(10^{6}\right)$ at a precession ratio comparable with $\mathrm{Po}^{\mathrm{c}}$ in our experiments at $\mathrm{Re}=10^{5}$. It seems likely that both phenomena are closely connected, with the $(m, k)=(0,2)$ mode being a precursor for the transition to the turbulent state observed in [32]. In the limit of large $\mathrm{Re}$ as they will occur in the liquid sodium experiment (up to $\operatorname{Re} \approx 10^{8}$ ), we thus expect dynamo action to arise in connection with the non-geostrophic axisymmetric mode within a width of $\Delta \mathrm{Po} \approx 0.006$ around $\mathrm{Po}$ not much smaller than $\mathrm{Po}^{\mathrm{c}}$ in our experiments at $\operatorname{Re}=10^{5}$.

Our results reveal a first promising - though narrow regime, defined by the presence of the axisymmetric mode, within which we expect dynamo action in the planned dynamo experiment. This is not a turbulent dynamo since there is no significant amount of turbulence as it would result, for example, from the resonant collapse reported in experimental studies of precessing flows with small nutation angles [33]. Our model rather constitutes a laminar dynamo driven by few large scale velocity modes, and our simulations and measurements indicate that time-dependent contributions remain weak even at the largest Re with the spectra always being determined by standing inertial waves. This is in contrast to the flow in the VKS dynamo where the fluctuations are of the same order as the mean flow. Instead, a comparison with the Riga Dynamo is more appropriate, in which a fully developed turbulence arises on top of a mean flow [34], and calculations based on the time-averaged flow field still provided good agreement with the experiment [35] proving that the turbulent $\beta$-effect remains negligible for such flows.

So far, we did not consider more realistic magnetic boundary conditions, like an insulating outer domain or the finite conductivity of the container made of stainless steel which will be focus of a future study. Preliminary results from models including a thin outer layer with the electrical conductivity reduced by a factor of 8 show an increment of $\mathrm{Rm}^{\mathrm{c}}$ by roughly $10 \%$ which is still well within the capabilities of the planned facility.

This study has been conducted in the framework of the project DRESDYN (DREsden Sodium facility for DYNamo and thermohydraulic studies) which provides the platform for the precession dynamo experiment at HZDR. The au- 
thors further acknowledge support by the Helmholtz Allianz LIMTECH and Bernd Wustmann for the mechanical design of the experiment.

*a.giesecke@hzdr.de

[1] J. Wicht and A. Tilgner, Space Sci. Rev. 152, 501 (2010)

[2] S. I. Braginsky and P. H. Roberts, Geophys. Astrophys. Fluid Dyn. 79, 1 (1995)

[3] C. C. Wu and P. H. Roberts, Geophys. Astrophys. Fluid Dyn. 107, $20(2013)$

[4] D. Cébron and R. Hollerbach, Astrophys. J. Lett. 789, L25 (2014)

[5] A. Tilgner, Phys. Fluids 17, 034104 (2005)

[6] C. A. Dwyer, D. J. Stevenson, and F. Nimmo, Nature (London) 479, 212 (2011); J. Noir and D. Cébron, J. Fluid Mech. 737, 412 (2013), B. P. Weiss and S. M. Tikoo, Science 346, 1198 (2014)

[7] W. V. R. Malkus, Science 160, 259 (1968), J. P. Vanyo, Geophys. Astrophys. Fluid Dyn. 59, 209 (1991)

[8] R. F. Gans, J. Fluid Mech. 41, 865 (1970), R. Manasseh, J. Fluid Mech. 243, 261 (1992), J. Fluid Mech. 315, 151 (1996), J. Noir, P. Cardin, D. Jault, and J.-P. Masson, Geophys. J. Int. 154, 407 (2003) R. Lagrange, P. Meunier, F. Nadal, and C. Eloy, J. Fluid Mech. 666, 104 (2011), S. Goto, A. Matsunaga, M. Fujiwara, M. Nishioka, S. Kida, M. Yamato, and S. Tsuda, Phys. Fluids 26, 055107 (2014), Y. Lin, J. Noir, and A. Jackson, Phys. Fluids 26, 046604 (2014), Y. Lin, P. Marti, and J. Noir, Phys. Fluids 27, 046601 (2015), F. Marques and J. M. Lopez, J. Fluid Mech. 782, 63 (2015), A. Giesecke, T. Albrecht, T. Gundrum, J. Herault, and F. Stefani, New J. Phys. 17, 113044 (2015), J. M. Lopez and F. Marques, Phys. Rev. Fluids 1, 023602 (2016), W. Mouhali, T. Lehner, J. Léorat, and R. Vitry, Exp. Fluids 53, 1693 (2012).

[9] M. Le Bars, D. Cébron, and P. Le Gal, Annu. Rev. Fluid Mech. 47, $163(2015)$

[10] J. Léorat, F. Rigaud, R. Vitry, and G. Herpe, Magnetohydrodynamics 39, 321 (2003); J. Léorat, 42, 143 (2006); F. Stefani, A. Gailitis, and G. Gerbeth, Zeitschrift für Angewandte Mathematik und Mechanik 88, 930 (2008)

[11] F. Stefani, S. Eckert, G. Gerbeth, A. Giesecke, T. Gundrum, C. Steglich, T. Weier, and B. Wustmann, Magnetohydrodynamics 48, 103 (2012).

[12] R. F. Gans, J. Fluid Mech. 45, 111 (1971)

[13] C.-C. Wu and P. Roberts, Geophys. Astrophys. Fluid Dyn. 103, 467 (2009), J. Ernst-Hullermann, H. Harder, and U. Hansen, Geophys. J. Int. 195, 1395 (2011); Y. Lin, P. Marti, J. Noir, and
A. Jackson, Phys. Fluids 28, 066601 (2016)

[14] A. Giesecke, T. Albrecht, G. Gerbeth, T. Gundrum, and F. Stefani, Magnetohydrodynamics 51, 293 (2015).

[15] C. Nore, J. Léorat, J.-L. Guermond, and F. Luddens, Phys. Rev. E 84, 016317 (2011), O. Goepfert and A. Tilgner, New J. Phys. 18, 103019 (2016)

[16] (), see Supplemental Material at [URL will be inserted by publisher] for animation of the three-dimensional structure of the axial flow at $\operatorname{Re}=10^{4}$ and $\mathrm{Po}=0.1$.

[17] H. M. Blackburn and S. J. Sherwin, J. Comp. Phys. 197, 759 (2004)

[18] A. Tilgner, Studia geoph. et geod. 42, 232 (1998).

[19] Y. Takeda, Int. J. Heat Fluid Flow 7, 313 (1986).

[20] T. Vogt, D. Räbiger, and S. Eckert, J. Fluid Mech. 753, 472 (2014)

[21] W. Thomson, Philos. Mag. 10, 152 (1880).

[22] H. P. Greenspan, The theory of rotating fluids (Cambridge University Press, 1968).

[23] X. Liao and K. Zhang, J. Fluid Mech. 709, 610 (2012)

[24] P. Meunier, C. Eloy, R. Lagrange, and F. Nadal, J. Fluid Mech. 599, 405 (2008)

[25] R. Monchaux, M. Berhanu, M. Bourgoin, M. Moulin, P. Odier, J.-F. Pinton, R. Volk, S. Fauve, N. Mordant, F. Pétrélis, A. Chiffaudel, F. Daviaud, B. Dubrulle, C. Gasquet, L. Marié, and F. Ravelet, Phys. Rev. Lett. 98, 044502 (2007)

[26] M. L. Dudley and R. W. James, Proc. R. Soc. Lond. A 425, 407 (1989), F. Ravelet, A. Chiffaudel, F. Daviaud, and J. Léorat, Phys. Fluids 17, 117104 (2005)

[27] H. P. Greenspan, J. Fluid Mech. 36, 257 (1969)

[28] F. H. Busse, J. Fluid Mech. 33, 739 (1968)

[29] A. Tilgner, Phys. Rev. Lett. 99, 194501 (2007).

[30] (), see Supplemental Material at [URL will be inserted by publisher] for animation of the three-dimensional structure of the magnetic energy density mapped with the azimuthal field.

[31] See Supplemental Material at [URL will be inserted by publisher] for flow structure from UDV measurements up to $\mathrm{Re}=$ $10^{5}$.

[32] J. Herault, T. Gundrum, A. Giesecke, and F. Stefani, Phys. Fluids 27, 124102 (2015)

[33] A. D. McEwan, J. Fluid Mech. 40, 603 (1970), R. Manasseh, J. Fluid Mech. 265, 345 (1994)

[34] A. Gailitis, G. Gerbeth, T. Gundrum, O. Lielausis, E. Platacis, and F. Stefani, C. R. Phys. 9, 721 (2008), 0807.0305.

[35] A. Gailitis, O. Lielausis, S. Dement'ev, E. Platacis, A. Cifersons, G. Gerbeth, T. Gundrum, F. Stefani, M. Christen, H. Hänel, and G. Will, Phys. Rev. Lett. 84, 4365 (2000); A. Gailitis, O. Lielausis, E. Platacis, G. Gerbeth, and F. Stefani, Phys. Plasmas 11, 2838 (2004) 